\title{
A CANONICAL TRACE CLASS APPROXIMANT
}

\author{
D. A. LEGG AND J. D. WARD
}

\begin{abstract}
Let $H$ be a finite-dimensional Hilbert space, $B(H)$ the space of bounded linear operators on $H$, and $C$ a convex subset of $B(H)$. If $A$ is a fixed operator in $B(H)$, then $A$ has a unique best approximant from $C$ in the $C_{p}$ norm for $1<p<\propto$. However, in the $C_{1}$ (trace) norm, $A$ may have many best approximants from $C$. In this paper, it is shown that the best $C_{p}$ approximants to $A$ converge to a select trace class approximant $A_{1}$ as $p \rightarrow 1$. Furthermore, $A_{1}$ is the unique trace class approximant minimizing $\sum_{i=1}^{n} S_{i}(A-B) \ln S_{i}(A-B)$ over all trace class approximants $B$. The numbers $S_{i}(T)$ are the eigenvalues of the positive part $|T|$ of $T$.
\end{abstract}

1. Introduction. The question of approximating a finite-dimensional matrix by operators from some prescribed convex set in the $C_{p}$ norm, $1<p<\infty$, has a simple answer. Namely, there is always a unique approximant due to the convexity of the class of approximating operators and the uniform rotundity of the $C_{p}$ norm. However, in general a given matrix has a "large" set of approximants in the $C_{p}$ norm for $p=1$ or $\infty$.

The purpose of this paper is to show that the unique $C_{p}$ approximants to a given finite-dimensional matrix $A$ from a prescribed convex set converge to a "select" $C_{1}$ approximant as $p$ tends to 1 . This result answers, in the affirmative, questions 3 and 4 in [8]. In addition, the fact that the limit exists as $p \rightarrow 1$ establishes a canonical trace class approximant. Very often canonical approximants shed much light on the structure of the set of best approximants as seen in [2, 3 and 4$]$.

2. A canonical trace class approximant. In what follows, the term operator shall refer to a bounded linear operator on a complex Hilbert space $H$ of dimension $n<\infty$. The numbers $S_{1}(A), \ldots, S_{n}(A)$ will denote the eigenvalues of $|A|$ listed in decreasing order, where $A$ is a bounded linear operator on $H$ having polar factorization $A=U|A|$. For $1 \leqslant p<\infty$, the $C_{p}$ norm is defined by

$$
\|A\|_{p}=\left(\sum_{i=1}^{n} S_{i}(A)^{p}\right)^{1 / p} .
$$

For a given matrix $A$, let $A_{p}^{\prime}$ denote a unique nearest point in the $C_{p}$ norm from a closed convex set $C$ and let $A_{p}=A-A_{p}^{\prime}$. We establish that $\lim _{p \rightarrow 1} A_{p}$ (and hence $\left.\lim _{p \rightarrow 1} A_{p}^{\prime}\right)$ exists in the trace norm.

Received by the editors June 28, 1984.

1980 Mathematics Subject Classificatıon. Primary 47A99; Secondary 41A65, 41A50, 47A30. 
Motivated by the approach in [7] we let, for a fixed $A$,

$$
\phi(A, p)=\|A\|_{p}^{p}=\sum_{i=1}^{n} S_{i}(A)^{p} .
$$

Then

$$
\frac{d \phi}{d p}(A, p)=\sum_{i=1}^{n} S_{i}(A)^{p} \ln S_{i}(A),
$$

and so

$$
\frac{d \phi}{d p}(A, 1)=\sum_{i=1}^{n} S_{i}(A) \ln S_{i}(A),
$$

where ln denotes the natural $\log$.

LEMMA 2.1. If $B$ is any cluster point of $\left\{A_{p}\right\}$ in the trace norm as $p \rightarrow 1$, then $A-B$ is a best $C_{1}$ approximant to $A$ from $C$, and

$$
\sum_{i=1}^{n} S_{i}(B) \ln S_{i}(B)=\min _{D \in D} \sum_{i=1}^{n} S_{i}(D) \ln S_{i}(D),
$$

where $\mathscr{D}=\left\{A-D^{\prime}: D^{\prime}\right.$ is a best $C_{1}$ approximant to $A$ from $\left.C\right\}$.

Proof. Let $p_{k} \rightarrow 1$ with $A_{p_{k}} \rightarrow B$ in the trace norm. Clearly $A-B$ is a best $C_{1}$ approximant. Let $\Phi(x)=x \ln x$ for $x>0$ with $\Phi(0)=0$. Let $D_{1} \in \mathscr{D}$ satisfy

$$
\sum_{i=1}^{n} \Phi\left(S_{i}\left(D_{1}\right)\right)=\min _{D \in \mathscr{D}} \sum_{i=1}^{n} \Phi\left(S_{i}(D)\right) \text {. }
$$

It is easily checked that $\Phi(x) \leqslant\left(x^{p}-x\right) /(p-1)$ for $x \geqslant 0, p>1$, and so, for each $i=1, \ldots, n$, we have

$$
\Phi\left(S_{i}\left(A_{p_{k}}\right)\right) \leqslant \frac{S_{i}\left(A_{p_{k}}\right)^{p_{k}}-S_{i}\left(A_{p_{k}}\right)}{p_{k}-1} .
$$

Hence

$$
\begin{aligned}
\sum_{i=1}^{n} \Phi\left(S_{i}\left(A_{p_{k}}\right)\right) & \leqslant \frac{1}{p_{k}-1}\left[\sum_{i=1}^{n} S_{i}\left(A_{p_{k}}\right)^{p_{k}}-\sum_{i=1}^{n} S_{i}\left(A_{p_{k}}\right)\right] \\
& \leqslant \frac{1}{p_{k}-1}\left[\sum_{i=1}^{n} S_{i}\left(D_{1}\right)^{p_{k}}-\sum_{i=1}^{n} S_{i}\left(D_{1}\right)\right] \\
& =\frac{1}{p_{k}-1}\left[\phi\left(D_{1}, p_{k}\right)-\phi\left(D_{1}, 1\right)\right] .
\end{aligned}
$$

Letting $k \rightarrow \infty$, we obtain

$$
\sum_{i=1}^{n} \Phi\left(S_{i}(B)\right) \leqslant \sum_{i=1}^{n} \Phi\left(S_{i}\left(D_{1}\right)\right) .
$$

Hence

$$
\sum_{i=1}^{n} \Phi\left(S_{i}(B)\right)=\min _{D \in \mathscr{D}} \sum_{i=1}^{n} \Phi\left(S_{1}(D)\right) .
$$


THEOREM 2.2 Let $C$ be a closed convex set of $n \times n$ matrices and let $A_{p}$ be the matrix in $C$ with smallest $C_{p}$ norm. Then $\lim _{p \rightarrow 1} A_{p}$ exists.

Proof. Since the underlying Hilbert space is finite dimensional, every sequence $\left\{A_{p_{k}}\right\}, p_{k} \rightarrow 1$, contains a convergent subsequence in the trace norm. Hence it suffices to show that $\left\{A_{p}\right\}$ has a unique cluster point as $p \rightarrow 1$.

Suppose $B_{1}$ and $B_{2}$ are cluster points of $\left\{A_{p}\right\}$ as $p \rightarrow 1$. If we multiply $B_{1}$ and $B_{2}$ by a sufficiently large positive scaling factor, we may assume that all the numbers

$$
S_{i}\left(B_{1} / 2\right), \quad S_{i}\left(B_{2} / 2\right), \quad S_{i}\left(\frac{1}{2}\left(B_{1}+B_{2}\right)\right),
$$

$i=1, \ldots, n$, are either 0 or greater than 2 .

Let $f(x)$ be a strictly increasing convex function such that $f(0)=0$ and $f(x)=$ $\Phi(x)$ for $x \geqslant 2$. By [5, Chapter 2, Theorem 4.1], we have

$$
\begin{aligned}
\sum_{i=1}^{n} \Phi\left(S_{i}\left(\frac{1}{2}\left(B_{1}+B_{2}\right)\right)\right) & =\sum_{i=1}^{n} f\left(S_{i}\left(\frac{1}{2}\left(B_{1}+B_{2}\right)\right)\right) \\
& \leqslant \sum_{i=1}^{n} f\left(S_{i}\left(\frac{B_{1}}{2}\right)+S_{i}\left(\frac{B_{2}}{2}\right)\right) \\
& \leqslant \sum_{i=1}^{n}\left[\frac{1}{2} f\left(S_{i}\left(B_{i}\right)\right)+\frac{1}{2} f\left(S_{i}\left(B_{2}\right)\right)\right] \\
& =\frac{1}{2} \sum_{i=1}^{n} \Phi\left(S_{i}\left(B_{1}\right)\right)+\frac{1}{2} \sum_{i=1}^{n} \Phi\left(S_{i}\left(B_{2}\right)\right) .
\end{aligned}
$$

Hence by Lemma 2.1 , since $B_{1}$ and $B_{2}$ are the cluster points of $\left\{A_{p}\right\}$ as $p \rightarrow 1$, we have

$$
\sum_{i=1}^{n} \Phi\left(S_{i}\left(\frac{1}{2}\left(B_{1}+B_{2}\right)\right)\right) \leqslant \min _{D \in \mathscr{D}} \sum_{i=1}^{n} \Phi\left(S_{i}(D)\right) .
$$

Since $\frac{1}{2}\left(B_{1}+B_{2}\right) \in \mathscr{D}$, it follows that

$$
\sum_{i=1}^{n} \Phi\left(S_{i}\left(\frac{1}{2}\left(B_{1}+B_{2}\right)\right)\right)=\min _{D \in \mathscr{D}} \sum_{i=1}^{n} \Phi\left(S_{i}(D)\right) .
$$

Hence both inequality signs in (1) and (2) must actually be equal signs.

The inequality (1) implies that

$$
S_{i}\left(\frac{1}{2}\left(B_{1}+B_{2}\right)\right)=S_{i}\left(B_{1} / 2\right)+S_{i}\left(B_{2} / 2\right)
$$

for each $i=1, \ldots, n$ since $f$ is strictly convex, while (2) implies that $S_{i}\left(B_{1}\right)=S_{i}\left(B_{2}\right)$ $=S_{i}\left(\left(B_{1}+B_{2}\right) / 2\right)$ for all $i$; i.e., $\left\|B_{1}\right\|_{p}=\left\|B_{2}\right\|_{p}=\left\|\left(B_{1}+B_{2}\right) / 2\right\|_{p}$. From the strict convexity of the $C_{p}$ norm, it follows that $B_{1}=B_{2}$. Hence there exists a unique cluster point of $\left\{A_{p}\right\}$ as $p \rightarrow 1$ and this completes the proof.

REMARK. A similar result seems likely in the case $p=\infty$ if the approximating class is the set of positive operators. We conjecture that the best $C_{p}$ approximants converge in the operator norm to the approximant $P_{m}$ as $p \rightarrow \infty$. See [8]. 


\section{REFERENCES}

1. R. H. Bouldin, Positive approximants, Trans. Amer. Math. Soc. 177 (1977), 391-403.

2. R. H. Bouldin and D. D. Rogers, Normal dilations and operator approximations, Acta Sci. Math. (Szeged) 39 (1977), 233-243.

3. C. K. Chui, P. W. Smith and J. D. Ward, Approximation with restricted spectra, Math. Z. 144 (1975), 289-297.

4. __ Favard's solution is the limit of $W^{h, p}$ splines, Trans. Amer. Math. Soc. 220 (1976), 299-305.

5. I. C. Gohberg and M. G. Krein, Introduction to the theory of linear nonselfadjoint operators, Transl. Math. Mono., Vol. 18, Amer. Math. Soc., Providence, R. I., 1969.

6. P. R. Halmos, Positive approximants of operators, Indiana Univ. Math. J. 21 (1971), 951-960.

7. D. Landers and L. Rogge, Natural choice of $L_{1}$-approximants, J. Approx. Theory 33 (1981), 268-280.

8. D. D. Rogers and J. D. Ward, $C_{p}$-minimal positive approximants, Acta Sci. Math. (Szeged) 43 (1981), $109-115$.

Department of Mathematics, Indiana University - Purdue University, Fort Wayne, Indiana 46805

Department of Mathematics, Texas A \& M University, College Station, Texas 77843 